\title{
Les initiatives citoyennes de soutien aux migrants en Belgique. De l'humanitaire à la contestation politique
}

Elsa Mescoli, Antoine Roblain et Pieter Griffioen

\section{(2) OpenEdition}

\section{Journals}

Édition électronique

URL : https://journals.openedition.org/anthropodev/1031

DOI : 10.4000/anthropodev. 1031

ISSN : 2553-1719

Éditeur

Presses universitaires de Louvain

Édition imprimée

Date de publication : 1 décembre 2020

Pagination : 171-185

ISBN : 978-2-39061-078-6

ISSN : 2276-2019

\section{Référence électronique}

Elsa Mescoli, Antoine Roblain et Pieter Griffioen, «Les initiatives citoyennes de soutien aux migrants en Belgique. De l'humanitaire à la contestation politique », Anthropologie \& développement [En ligne], 51 | 2020, mis en ligne le 01 juin 2021, consulté le 28 juin 2022. URL : http://journals.openedition.org/ anthropodev/1031; DOI : https://doi.org/10.4000/anthropodev.1031

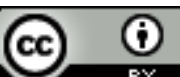

La revue Anthropologie \& développement est mise à disposition selon les termes de la Licence Creative Commons Attribution 4.0 International. 


\title{
Les initiatives citoyennes de soutien aux migrants en Belgique
}

\author{
De I'humanitaire à la contestation politique
}

Elsa Mescoli ${ }^{1}$, Antoine Roblain ${ }^{2}$ et Pieter Griffioen ${ }^{3}$

\begin{abstract}
À l'instar de nombreux pays européens, la Belgique fait face depuis 2015 à l'arrivée de migrants marqués par une extrême vulnérabilité. Leur accueil a amené les sociétés européennes à se polariser autour de la question migratoire et a suscité de larges débats portant sur la gestion sécuritaire des migrations et les réponses humanitaires à apporter. Dans ce contexte, la Belgique a vu fleurir de nombreuses initiatives citoyennes désirant combler les manquements (perçus ou réels) des institutions d'accueil et d'intégration. Construites et structurées autour de l'aide humanitaire à pourvoir aux migrants, ces initiatives participent à l'émergence de dynamiques (et demandes) relationnelles dépassant la « simple » réponse aux besoins primaires et elles déclenchent des positionnements et des revendications d'ordre politique. Sur la base de deux études de cas engageant principalement des migrants provenant de pays africains, cet article présente une analyse micro des processus par lesquels les relations tissées au sein de l'action humanitaire participent à la redéfinition des finalités de ces mouvements citoyens, faisant entrer des objectifs politiques dans une action centrée sur un registre initialement éthique. Notre analyse s'adresse également aux espaces et formes de prise de parole des migrants au sein de ces dynamiques, et du processus de leur politisation.
\end{abstract}

As in many European countries, Belgium, since 2015, has faced the arrival of migrants marked by extreme vulnerability. Their reception has led European societies to shape polarized opinions around the issue of migration and has triggered large debates on the management of migration through a securitization approach, as well as the humanitarian responses to it. In this context, Belgium has witnessed the appearance of a number of citizens' initiatives wishing to supplement the failings (perceived or real) of reception and integration institutions. Constructed and structured around humanitarian aid to be provided to migrants, these initiatives contribute to the emergence of relational dynamics (and demands) that go beyond the "simple" response to primary needs, and trigger political positions and claims. Based on two case studies mainly engaging migrants coming from African countries, this article brings a micro-analysis of the processes through which the relations within humanitarian action contributes to the redefinition of the goals of these citizens' movements, bringing political objectives into an action originally focused on an ethical register. Our analysis also addresses the spaces and forms of speaking up by migrants within these dynamics, and the process of their politicization.

\footnotetext{
${ }^{1}$ Anthropologue, université de Liège, centre d'études de l'ethnicité et des migrations (CEDEM), laboratoire d'anthropologie sociale et culturelle (LASC) ; e.mescoli@uliege.be

2 Psychologue social, université libre de Bruxelles, centre de recherche en psychologie sociale et interculturelle (CeSCuP) ; aroblain@ulb.ac.be

${ }^{3}$ Chercheur, université libre de Bruxelles, Group for Research on Ethnic Relations, Migration and Equality (GERME) ; pieter.h.griffioen@googlemail.com
} 


\section{Introduction $^{4}$}

Les trente dernières années ont vu l'expression de plus en plus affirmée d'un tournant au sein des politiques migratoires européennes, considérant la mobilité humaine vers les pays occidentaux comme indésirable et menaçante (Valluy, 2009). Liée à un imaginaire d'envahissement, la mobilité de ces indésirables vers l'Europe est jalonnée de dispositifs visant à les contrôler et les repousser, à les criminaliser et les enfermer. Néanmoins, la sécurisation de la migration se confronte à une autre orientation au sein des discours et des politiques qui tend à considérer le migrant comme une victime dans le besoin, une personne vulnérable en attente de soutien humanitaire. Intimement liée à la perception du corps souffrant, cette représentation renvoie les sociétés européennes à leur " raison humanitaire » (Fassin, 2010) et elle déclenche la mobilisation des individus.

En Belgique, à l'instar d'autres pays européens, la période dite de " crise " (des réfugiés, de la migration ou de l'accueil selon les perspectives adoptées) de 2015 a été marquée par la rencontre et la confrontation de ces deux tendances. D'une part, la sécurisation et la criminalisation des migrations agissent notamment par des pratiques de surveillance et des modifications législatives impactant la durée et le droit au séjour ${ }^{5}$. D'autre part, la raison humanitaire se manifeste à travers l'émergence de nombreux mouvements citoyens de soutien aux migrants sur le sol européen (Rosenberger et al., 2018).

Cet article tend à démontrer que, si ces mouvements citoyens se construisent autour de valeurs morales de l'hospitalité, leurs actions s'inscrivent également dans le registre du politique. En partant d'analyses ethnographiques de deux initiatives citoyennes apportant un soutien à des migrants majoritairement africains en situation irrégulière en Belgique, il s'agit ici d'appréhender les formes de contestation des politiques sécuritaires émergeant de mouvements citoyens. À cet égard, nous soutenons que le passage de l'activisme humanitaire au politique se fait à un niveau micro, au sein des relations interpersonnelles engageant migrants et agents humanitaires (professionnels ou non). Ce processus contribue à la formation d'une conscience collective partagée et transforme l'engagement

\footnotetext{
${ }^{4}$ Elsa Mescoli et Antoine Roblain sont coauteurs de cet article, ils ont contribué de manière équivalente à son écriture. Pieter Griffioen a contribué à la réflexion en amenant des matériaux ethnographiques concernant le terrain de Bruxelles.

${ }^{5}$ À titre d'exemple, la loi du 17 mai 2016 modifiant les articles 10ter et 12bis de la loi du 15 décembre 1980 sur l'accès au territoire, le séjour, l'établissement et l'éloignement des étrangers allonge le délai de traitement des demandes de regroupement familial. Suite à la loi du $1^{\text {er }}$ juin 2016, les réfugiés reconnus en Belgique ne se voient plus accorder immédiatement un droit de séjour d'une durée illimitée, mais d'abord un titre de séjour temporaire (voir : https://www.cgra.be/fr/actualite/refugies-reconnus-mise-jour-de-labrochure-suite-la-modification-de-la-loi, consulté le 3 avril 2019). En ce qui concerne les procédures de régularisation pour des "circonstances exceptionnelles ", il n'existe pas de critères clairs et " une large place est laissée au pouvoir et à l'appréciation de l'Administration (Office des étrangers) " (voir : https://www.vivreenbelgique.be/sejour-en-belgique/la-regularisation, consulté le 3 avril 2019), ce qui détermine une diminution continue des autorisations de séjour définitif (selon le Centre fédéral Migration, Myria, voir : https://www.myria.be/fr/publications/la-migration-en-chiffres-et-en-droits-2018, consulté le 3 avril 2019).
} 
humanitaire en acte de solidarité politique. En d'autres mots et dans la lignée de Martina Tazzioli et William Walters (2019), nous montrerons comment les gestes principalement individuels de solidarité vont finalement s'ancrer dans des luttes de pouvoir sociales et politiques au sein de la société.

Suivant un encadrement théorique des réflexions et une contextualisation des matériaux collectés et analysés, nous étudions les modalités d'émergence d'une orientation politique au sein de mouvements citoyens de soutien humanitaire aux migrants. L'attention est portée sur les pratiques et les discours mis en œuvre par les membres de la Plateforme citoyenne de soutien aux réfugiés à Bruxelles et de la Voix des sans-papiers de Liège, avec pour objectif de montrer que les relations qui se nouent entre l'aidant et l'aidé dans le cadre humanitaire participent à l'émergence d'une conscience politique chez les acteurs concernés (y compris, dans certains cas, les migrants). Par l'étude de deux exemples concrets du déploiement d'un processus de politisation de logiques humanitaires, cet article ambitionne ainsi de complexifier le discours porté sur les migrants, allant au-delà de la polarisation entre la logique sécuritaire et la logique humanitaire et proposant une approche réhabilitant les individus en tant que détenteurs de droits.

\section{La nature des relations dans le cadre humanitaire}

Touchant particulièrement "les personnes de couleur " et, de manière disproportionnée, les ressortissants d'Afrique subsaharienne vus comme les plus indésirables parmi les migrants (De Genova, 2018 : 1767), le contrôle sécuritaire des mouvements migratoires se manifeste dans les contextes d'arrivée par une menace et une surveillance constantes menées - visiblement ou invisiblement - par les forces de l'ordre (Brennan, 2018) et les gouvernements. Les initiatives citoyennes de soutien aux migrants s'opposent aux rhétoriques favorisant l'orchestration d'un consensus (Kosnick, 2019 : 174) autour de l'indésirabilité des migrants. Elles vont également à l'encontre des dispositifs politiques d'exclusion des migrants basés sur des vecteurs d'émotions telles que la peur et la colère, ou l'« emo-politis » (Nussbaum, 2018). Défiant une panique morale largement diffusée, ces initiatives établissent une configuration éthique fondée sur la compassion (Ticktin, 2006).

Néanmoins, la littérature contemporaine portant sur l'aide humanitaire, incluant l'aide orientée vers les réfugiés et les demandeurs d'asile, a produit de nombreux travaux soutenant que ces interventions ont tendance à se muer en dispositifs de gouvernementalité (Agier, 2008 ; Fassin, 2010 ; Pandolfi, 2000). L'aide humanitaire aurait cette inclination à instaurer ou maintenir des hiérarchies de pouvoir entre le " monde " et les " restes du monde " (Agier, 2008), entre les institutions et les sujets "indésirables ", tels que les migrants. L'aide humanitaire apparaît ainsi comme un cadre contraignant l'agentivité des migrants et comme un dispositif de mise à l'écart de ces derniers (Agier, 2008 ; Siméant, 1998). Comme le soulignait déjà Jean-Pierre Olivier de Sardan (1975), ce dispositif d'éloignement est à l'œuvre dans les rapports sociaux qui se déploient à l'intérieur de la relation humanitaire. Plus récemment, Barbara Harrell-Bond (2002), réactualisant la notion de don et contre-don élaborée par Marcel Mauss (1923), soutient que le travail 
humanitaire fait entrer l'agent humanitaire et les migrants dans une relation profondément asymétrique. Les enjeux complexes de réciprocités qui se jouent au sein de la relation d'aide impliquent tant des attentes que des frustrations chez les acteurs concernés (Heins et Unrau, 2018). L'action humanitaire, en tant que dispositif d'une volonté gouvernementale visant la perpétuation de déséquilibres relationnels et sociopolitiques, priverait a priori certains individus de la possibilité de s'engager activement dans un processus de prise de parole, en contraignant leur initiative au seul domaine et régime de gestion de corps souffrants (Ticktin, 2006 ; Agamben, 1998).

Cependant, l'analyse micro des initiatives humanitaires par le biais des redéfinitions des relations interpersonnelles qui s'y jouent permet de mettre en lumière des pratiques de contestation et des processus de prise de parole politique basés sur la revendication de droits. Les migrants retrouvent une place d'acteurs et élaborent une subjectivité politique propre en rétablissant une réciprocité de l'échange (Phillimore et al., 2018) ou en affirmant une autonomie d'action et de parole, des "capacités à contourner ou à s'approprier peu ou prou les incitations auxquelles ils étaient soumis » (Atlani-Duault et Dozon, 2011: 397). Comme le suggère Martin Deleixhe (2018), la rencontre entre volontaires humanitaires et migrants va servir de levier favorisant le passage d'une mobilisation centrée sur le registre de la morale et de l'éthique vers des contestations politiques amenant à reconnaître le migrant non seulement comme une personne à aider, mais également comme un sujet de droit. Les revendications faites au départ de l'intervention humanitaire vont de la sollicitation à la mise en place d'une politique d'accueil " humaine » qui s'engage dans le respect des droits fondamentaux, à des actions plus larges de citoyenneté (Isin et Nielsen, 2013) incluant, entre autres, une gestion inclusive des enjeux migratoires.

Finalement, l'interconnexion entre morale et politique (Fassin, 2007) se manifeste dans un apparent paradoxe: d'un côté la résistance humanitaire à la sécurisation de la migration risque de servir de nouvelles formes de gouvernementalité qui empêcheraient l'engagement politique des acteurs concernés, et d'un autre côté elle contient un potentiel de subversion de cet état de choses (Vandevoordt et Verschraegen, 2019). L'objectif de cet article est de montrer que ce passage entre morale et politique se fait au sein des initiatives citoyennes au travers d'une redéfinition et d'une négociation des relations entretenues par les agents humanitaires et les migrants.

\section{Ethnographie de l'aide humanitaire aux migrants}

Les initiatives citoyennes d'aide aux migrants étudiées dans cet article sont situées l'une dans la ville de Bruxelles, l'autre dans la ville de Liège. Un travail ethnographique y a été mené entre novembre 2017 et décembre 2018, mêlant des observations participantes des activités mises en place, une recherche documentaire sur les principales plateformes de conversation des bénévoles, et des entretiens individuels avec les personnes clés des mouvements et des associations œuvrant aux côtés de ces initiatives. À Bruxelles, quarante entretiens individuels ont également été réalisés avec les migrants, tandis qu'à 
Liège les discours de ces interlocuteurs ont été collectés via l’observation participante des initiatives dans lesquelles ils étaient engagés.

Les deux populations migrantes de ces deux terrains de recherche se rejoignent en ce qui concerne leur statut juridique, à savoir l'irrégularité de séjour des individus. Néanmoins, ces migrants s'inscrivent dans des carrières migratoires (Martiniello et Rea, 2014) profondément distinctes, amenant des histoires et des projets individuels et collectifs diversifiés.

Les migrants présents à Bruxelles sont généralement arrivés depuis peu sur le sol belge et $s^{\prime} y$ inscrivent pour la plupart dans une temporalité relativement courte. Peu d'entre eux souhaitent introduire des demandes de régularisation (des demandes d'asile le cas échéant) auprès des autorités belges dans la mesure où ils considèrent la Belgique comme un lieu de transit vers le Royaume-Uni. Aucune statistique officielle concernant le nombre et les caractéristiques de cette population n'existe. Néanmoins, un récent rapport de Médecins sans frontières (2019) donnant des informations sur les migrants passés par leur permanence médicale entre septembre 2017 et septembre 2018 nous permet d'avoir un regard sur leurs caractéristiques sociodémographiques. Ce rapport indique que la grande majorité des migrants est de genre masculin (97\%), que $13 \%$ d'entre eux étaient mineurs lors de leur visite et que la tranche d'âge allant de 19 à 45 ans est majoritaire. Enfin, les principales nationalités représentées sont les pays de la Corne de l'Afrique ainsi que le Soudan ; viennent ensuite certains pays du Maghreb, l'Égypte, la Libye, l'Afghanistan et la Syrie.

À l'opposé, la trajectoire de vie des « sans-papiers " ${ }^{6}$ " présents au sein du mouvement de Liège témoigne de la volonté d'une résidence permanente sur le territoire. Présents depuis jusqu'à dix ans en Belgique, ces individus, outre dans des initiatives de mobilisation, sont souvent engagés dans des activités professionnelles dans l'économie informelle, ou dans des stages ${ }^{7}$; les enfants du collectif sont scolarisés, certains adultes suivent également des études supérieures. La composition du groupe est hétérogène, incluant jusqu'à 27 nationalités ${ }^{8}$, dont le Congo, le Rwanda, la Guinée, le Niger, le Sénégal, le Burkina Faso, le Cameroun, l'Ouganda, le Bénin, le Togo. Le nombre de personnes inscrites dans ce mouvement peut être difficilement établi, parce que, si une centaine de migrants au total vivent dans deux occupations principales du collectif, avec une légère majorité d'hommes et des âges diversifiés (de l'enfance jusqu'aux 67 ans du membre le plus âgé), $d$ 'autres sont affiliés au mouvement tout en résidant ailleurs (auprès de connaissances ou dans la rue).

\footnotetext{
${ }^{6}$ Conscients de la portée de ce terme, nous le reprenons tout au long de cet article en tant que notion émique émergée durant l'activité de terrain et catégorie réappropriée de la part des migrants eux-mêmes comme point de départ de leurs actions de mobilisation.

${ }^{7}$ L'irrégularité de séjour n'empêche pas l'inscription et le suivi de ces formations, néanmoins elle détermine l'impossibilité d'acquérir un diplôme et d'être rétribué (dans le cas des stages).

${ }^{8}$ Voir : https://www.lavenir.net/cnt/dmf20150821_00690497 (consulté le 3 avril 2019).
} 
Les populations migrantes de ces deux terrains, ainsi que les autres acteurs engagés dans les initiatives en question, se positionnent dans les contextes étudiés de manière différenciée, générant des conceptions propres de l'aide humanitaire. Nous l'entendons globalement comme l'ensemble de pratiques et discours orientés vers la réponse aux besoins primaires des êtres humains dans des contextes au sein desquels, pour des raisons diverses, cette réponse n'est pas fournie par les institutions gouvernementales. Cette aide vise en premier lieu l'hébergement, et s'ouvre par la suite à une série d'autres actions diversifiées selon les contextes, allant de la préparation de repas au don de vêtements et de matériels divers (cartes pour téléphones mobiles, produits de soin, matériel scolaire pour les enfants, ustensiles ménagers, etc.). Pareillement, les deux initiatives formulent des revendications distinctes: du droit à la mobilité dans des conditions respectueuses des êtres humains, au droit à la résidence et à l'inclusion dans le contexte d'arrivée. Les deux groupes n'étant pas en communication directe entre eux ${ }^{9}$, ils promeuvent des initiatives autonomes et aux contenus en partie différents, ce qui détermine aussi des divergences quant à la formation d'une conscience politique collective et à la volonté de prise de parole des migrants. Néanmoins, les deux contextes étudiés fournissent des exemples parallèles de l'émergence d'une orientation politique au sein des initiatives humanitaires, et ils permettent d'éclairer ce processus via l'analyse des relations qui y sont établies, négociées et redéfinies.

\section{Discussion}

\section{La Plateforme citoyenne de soutien aux réfugiés et ses volontaires hébergeurs : de la rencontre humanitaire à la contestation politique}

Durant l'été 2017, un des quartiers les plus administratifs de Bruxelles, en plein centreville, a vu une augmentation substantielle du nombre de migrants venant, passant, attendant et errant avec pour premier objectif de trouver un moyen de rejoindre le Royaume-Uni. N'étant pas inscrits dans le système d'accueil pour requérants d'asile organisé par l'État fédéral, ces migrants posaient une série de problématiques d'ordre humanitaire. La réponse du gouvernement face à cette situation a été sécuritaire, avec la mise à distance de ces migrants ne répondant à aucune case administrative. L'organisation de descentes policières dans le quartier a mené à l'arrestation et à la dispersion des personnes en situation irrégulière: "environ vingt à trente personnes sont arrêtées chaque jour dans les activités quotidiennes » (procès-verbal d'une réunion de préparation aux actions policières, 4 septembre 2017).

\footnotetext{
${ }^{9}$ La Voix des sans-papiers de Liège est bien un collectif initié en rapport aux actions de la Coordination des sans-papiers de Belgique située à Bruxelles. Cette dernière a entretenu des relations - et des tensions avec certains membres et initiatives de la Plateforme de soutien aux réfugiés. Ce n'est pas le cas par contre pour la Voix des sans-papiers de Liège, dont les démarches se situent majoritairement dans le contexte local, au sein duquel aucun regroupement important de personnes en transit ne se trouve, ce qui détermine l'absence de croisements ou oppositions concrets des intérêts et des revendications.
} 
Brandissant le spectre de l'appel d'air et utilisant la situation de Calais comme repoussoir, les responsables politiques au niveau fédéral se sont radicalement opposés à la prise en charge des migrants présents sur le territoire et ont négocié et conclu des accords avec certains pays africains pour faciliter les expulsions. Cette position politique est plus particulièrement portée par Theo Francken, ex-secrétaire d'État à l'Asile et à la Migration :

Arrêter de renvoyer au Soudan, alors que tous nos pays voisins continuent de le faire, risque de générer un énorme effet d'appel d'air en Belgique de personnes soudanaises en résidence illégale. [...] Et nous avons vu dans le Nord de la France ce que cela peut entraîner. (traduction libre d'un message posté sur le blog officiel, 22 décembre 2017) ${ }^{10}$

En réponse à ces politiques, un collectif de citoyens - la Plateforme citoyenne de soutien aux réfugiés, constituée en 2015 au moment de l'émergence des problématiques de l'accueil de demandeurs d'asile majoritairement syriens - s'est à nouveau mobilisé pour trouver des solutions d'hébergement pour ces migrants et leur permettre d'échapper aux arrestations. Profitant d'un cadre légal permettant l'exercice d'une aide aux personnes en situation irrégulière pour des raisons principalement humanitaires ${ }^{11}$, cette initiative a rapidement pris une ampleur importante et des réseaux de volontaires se sont organisés à travers l'ensemble du pays. Depuis l'été 2017, chaque soir, les membres de la coordination de la plateforme se retrouvent pour mettre en relation des migrants avec des volontaires se proposant d'ouvrir leur foyer pour une ou plusieurs nuits. Le quartier de la Gare du Nord est devenu le point de rendez-vous pour 200 à 300 migrants et leurs hébergeurs. Une fois le jour levé, ces migrants se retrouvent une nouvelle fois dans ce quartier et autour de cette gare routière pour tenter leur "chance ", selon leur propre terme, de rejoindre le Royaume-Uni.

Outre l'organisation de cet hébergement dans les foyers de volontaires, la plateforme citoyenne, dépassée par le nombre de migrants et voyant l'hiver s'installer, a ouvert le 11 décembre 2017, grâce à des financements publics régionaux, un centre d'accueil de nuit, baptisé Porte d’Ulysse. La capacité de ce centre est passée de 80 places lors de l'ouverture, à 200 et puis à 300 lits disponibles, à la suite du déménagement dans un bâtiment adjacent en 2018.

Parallèlement à l'aide à l'hébergement, la plateforme citoyenne a également développé d'autres activités parmi lesquelles un pôle politique et plaidoyer, prenant une place tout aussi inattendue qu'importante au sein de la contestation des politiques migratoires. La plateforme remet en question les conditions d'accueil des migrants et plus globalement les politiques sécuritaires jugées inhumaines :

La Plateforme citoyenne de soutien aux réfugiés veut construire une solidarité concrète avec tou.tes les migrant·es. Elle dénonce et veut combattre les politiques migratoires

\footnotetext{
${ }^{10}$ Voir : https://theotuurt.wordpress.com/2017/12/21/soedan-2/ (consulté le 8 avril 2019).

${ }^{11}$ Article 77 de la loi du 15 décembre 1980 sur les étrangers, voir : http://www.ejustice.just.fgov.be/ (consulté le 8 avril 2019).
} 
belges et européennes actuelles et rappelle que le droit de vivre dans la dignité appartient à tou.tes. (message de présentation sur le site web de la plateforme) ${ }^{12}$

Dans le prolongement de leur activisme humanitaire, les leaders du mouvement ont ainsi fait appel aux volontaires pour qu'ils deviennent les porteurs d'un message politique : «Mesdames, messieurs, vous avez votre place dans le débat. Vous avez le droit de vous exprimer et d'obtenir des réponses ", ce sont les mots d'un coordinateur de la plateforme (entretien, 2 mars 2018). Cette dimension politique s'est également manifestée par la participation de certains membres de la plateforme à l'organisation de nombreuses interpellations citoyennes au niveau communal. Parmi celles-ci, une motion contrant un projet de loi déposé par le gouvernement fédéral qui autoriserait les « visites domiciliaires " visant à interpeller des personnes en situation irrégulière dans les domiciles privés ${ }^{13}$. Comme le souligne la directrice de Coordination et initiatives pour réfugiés et étrangers (CIRE), " il y a eu basculement des citoyens qui étaient mobilisés dans la plateforme vers les collectifs citoyens actifs au niveau local concernant les étrangers " (entretien, 19 novembre 2018). Ce basculement s'est aussi manifesté par l'investissement des volontaires dans la campagne "Communes hospitalières " développée en 2017 par une coalition d'organisations dont fait notamment partie le CNCD11.11.11, le CIRE ou encore les syndicats ${ }^{14}$. Outre l'engagement de ses volontaires dans les initiatives portées par d'autres organisations, les coordinateurs de la plateforme ont entretenu des relations avec les acteurs cités ci-dessus dans le but de communiquer sur les activités tant humanitaires que politiques, instaurant des collaborations formelles et informelles, non exemptes néanmoins de tensions liées à l'émergence d'un nouvel acteur. Il est également à noter l'absence presque totale des migrants aidés par la plateforme citoyenne dans ces mouvements de revendication politique. Probablement liée à la caractéristique de leur carrière migratoire marquée par l'incertitude quant à leur présence sur le territoire belge, cette volonté d'invisibilité se dessine à l'inverse de la détermination à se rendre visibles et à légitimer une présence - stable - qui figure parmi les objectifs des membres du collectif liégeois.

Pour comprendre cette réorientation de l'aide humanitaire vers l'action politique, nous étudions la nature des relations entretenues entre volontaires et migrants.

\footnotetext{
${ }^{12}$ Voir : http://www.bxlrefugees.be/qui-sommes-nous/ (consulté le 8 avril 2019).

${ }^{13}$ Ce projet de loi déposé le 7 décembre 2017 visait à modifier la loi du 15 décembre 1980 sur l'accès au territoire, le séjour, l'établissement et l'éloignement des étrangers afin de garantir l'exécution des mesures d'éloignement. Jusqu'à présent, le projet de loi n'a pas eu de suites. Voir : http://www.lachambre.be/FLWB/PDF/54/2798/54K2798001.pdf (consulté le 8 avril 2019).

${ }^{14}$ Cette campagne avait pour objectif d'amener les citoyens à interpeller leurs élus locaux pour qu'ils mettent en place des dispositifs luttant contre la discrimination des migrants (empêchant par exemple l'arrestation de personnes en situation irrégulière) et facilitant l'accès à certains services (voir: https://www.communehospitaliere.be, consulté le 8 avril 2019).
} 


\section{À travers la rencontre, la reconnaissance de l'Autre comme un sujet de droit}

Dans la lignée des arguments développés par Martin Deleixhe (2018), si le volontaire humanitaire ancre dans un premier temps son acte dans le registre de la morale et de l'éthique, la rencontre de cet Autre en besoin est susceptible d'amener à le reconnaître non seulement comme un proche, mais également comme un sujet de droit. Un coordinateur de la plateforme décrit cette transition en valorisant l'effet obtenu, plutôt que de se positionner de manière critique par rapport au processus :

[...] quand les autres se plaignent en disant que le froid mobilise plus que la politique migratoire, je dis : " qu'importe que ce soit le froid, on s'en fout, ce n'est pas important ce qui t'a poussé à passer à l'acte, une fois que t'es passé à l'acte, t'es mordu ". (entretien, 2 mars 2018)

Selon lui, il s'agit d'une conscientisation des personnes engagées qui vont, ainsi, " continuer à en parler », impactant la société entière. Cette conscientisation passe par l'établissement de liens entre volontaires et migrants basés sur des dynamiques de réciprocité défiant les hiérarchies de pouvoir et les asymétries relationnelles imposées a priori par l'aide humanitaire. La solidarité émerge ainsi comme dispositif d'alliances horizontales (Tazzioli et Walters, $2019: 6$ ).

Cependant, cette réciprocité se décline sous plusieurs formes et soulève de nombreux débats au sein des volontaires de la plateforme. Une majorité des migrants rencontrés ont stipulé qu'ils ne ressentent aucune ou très peu d'attentes envers eux, et le discours officiel de l'initiative est celui d'un humanitaire où nulle réciprocité ne doit être attendue. Néanmoins des manifestations d'autres normes informelles instituant une attente de réciprocité sont également observables. Que cela soit explicite ou implicite, de nombreux hébergeurs nourrissent un imaginaire concernant leur rencontre avec cet Autre transitant en Belgique, son histoire et sa culture. En émerge une pression normative poussant les migrants, surtout lorsqu'ils sont hébergés dans les foyers, à partager leurs histoires et à faire vivre à leurs hôtes une expérience interculturelle :

[...] sometimes they want to see how we act. If we are honest, kind, cooperating. They see how your culture is, behaving good. If you are respectful, tell them your story, next time when they come they take you again. So they want to help you if you are a good person and respect them. They always ask us one question: Why you go to England? (migrant Somalien, entretien, 14 mars 2019)

Le partage de l'histoire de vie rétablit l'équilibre de l'échange et permet la perpétuation de la relation (réciproque) d'aide. Outre la rencontre d'un Autre culturellement différent, une frange importante des volontaires ont ouvert ces interactions à la construction de relations profondes et intimes. Cette tendance se manifeste dans les discours mobilisés par ces individus où les champs lexicaux de l'amour et de la parentalité sont largement utilisés pour décrire la relation qui les unit aux migrants :

M. est en prison et les nouvelles ne sont pas bonnes. T. est à l'hôpital et les nouvelles ne sont pas terribles. F. est en Angleterre et s'est enfui de son " camp " parce qu'on ne lui donnait pas à manger. M. y est aussi. [...] On se dit qu'on s'aime. II m'appelle " $m y$ mother ». Je souris à l'intérieur. [...] Alors, donc, je vais remplir ma maison de petits 
nouveaux. Je suis prête même si j'ai un peu peur. J'ai un peu peur de me mettre à les aimer eux aussi... (volontaire anonyme) ${ }^{15}$

Loin de faire l'unanimité au sein de la plateforme citoyenne, ces comportements sont questionnés par certains volontaires qui dénoncent l'usage excessif du pathos, une " sensiblerie larmoyante » qui dévierait des objectifs de l'initiative :

Nous n'aidons pas un réfugié parce qu'il est " un petit gars ", " brillant », " courageux » et "sympathique ", autrement dit "mignon", comme s'il s'agissait d'adopter un hamster. Nous le faisons parce qu'il s'agit d'un être humain en détresse. (bénévole de la plateforme, 20 janvier 2019) ${ }^{16}$

Ce glissement vers l'émancipation des asymétries inhérentes à l'action humanitaire se fait au sein des relations entre les migrants et les bénévoles de la plateforme citoyenne grâce à ces dynamiques de réciprocité. Ce qui permet aussi le passage de l'humanitaire à l'action politique, de l'aide vers l'Autre en besoin à sa reconnaissance comme un sujet de droit.

\section{La Voix des sans-papiers de Liège et le comité de soutien : activités et relations}

En interrogeant la relation aidant/aidé aussi au sein du terrain liégeois, nous analysons un autre exemple d'émergence d'une orientation politique basée sur la revendication de droits au sein d'un mouvement au départ humanitaire. Le collectif la Voix des sans-papiers de Liège (VSP) a été créé en 2015 à l'initiative d'un groupe de migrants en séjour irrégulier sur le territoire qui ont, pour la plupart, obtenu une réponse négative à leur demande d'asile. Leur action a commencé par l'occupation d'un bâtiment inhabité dans la périphérie de Liège. Dans la lignée des mouvements de longue date venant en aide aux sans-papiers de la ville, le Comité de soutien aux sans-papiers de Liège s'est regroupé pour apporter dans un premier temps de l'aide humanitaire centrée sur l'accommodation de ce logement :

Les premiers jours les « soutiens " [les membres du comité de soutien] se sont attelés à amener à manger, des bénévoles se sont mis à raccorder l'eau, à mettre l'électricité, à mettre des bouilloires, ils ont fait tout ce qu'il fallait pour occuper la maison. (ancien membre de la VSP, entretien, 30 janvier 2018)

À la suite de l'occupation d'un nouveau bâtiment arrivée peu après, une série d'autres activités ont été développées par le comité de soutien pour répondre à l'exclusion de ces migrants et à la volonté de créer des espaces où ils peuvent « sortir de leur quotidien et de leurs problèmes " (membre du comité de soutien, notes de terrain,

\footnotetext{
15 II s'agit d'un site internet regroupant une série de témoignages venant de bénévoles hébergeant des migrants : http://www.perlesdaccueil.be/2019/01/18/2520/ (consulté le 8 avril 2019).

${ }^{16}$ Ce message a été posté sur le groupe Facebook "Hébergement Plateforme citoyenne " organisant I'hébergement des migrants: https://www.facebook.com/groups/hebergementplateformecitoyenne/ (consulté le 8 avril 2019).
} 
22 novembre 2017). Ces activités alimentent une cause collective et permettent aux migrants de (re)devenir des sujets actifs :

[...] dans le cadre de l'approche sécuritaire qui finit par viser les personnes issues de la migration, des projets renforçant les compétences de celles-ci, indépendamment de leur statut, [...] sont mis en place. (membre d'une association locale, notes de terrain, 29 novembre 2017)

II s'agit notamment d'activités artistiques (ateliers d'écriture et de théâtre, peinture, cuisine, couture) et professionnelles (stages, formations) investissant des espaces publics, des locaux d'associations ou d'institutions locales - les salles de théâtre, les espaces syndicaux, entre autres - qui font fonction de lieu où la lutte politique s'exerce. Ces initiatives visent également à faire évoluer les représentations véhiculées au sein de la société par des politiques jugées discriminatoires à l'encontre, plus particulièrement, des migrants africains :

[...] le souci est de montrer aux gens qu'on n'est pas là à manger et dormir, mais on bouge et on sort la tête de l'eau. [...] Nous sommes gênés de voir à travers les médias [qu'on nous décrit comme] paresseux, dangereux. (membre de la VSP, notes de terrain, 22 janvier 2017)

Les agents humanitaires (membres de la société civile locale, incluant des professionnels comme les travailleurs des associations, des institutions, des syndicats, mais aussi des volontaires ne faisant partie d'aucun cadre structuré) facilitent cette démarche, faisant émerger chez les migrants un sentiment de redevabilité qui s'est souvent manifesté par l'expression publique de remerciements envers les personnes qui se mobilisent autour du groupe. L'asymétrie de départ de la relation entre les "soutiens » et les " sans-papiers » détermine l'inhibition relative à l'expression d'expectatives et d'éventuels désaccords de la part des migrants, tandis qu'il est très fréquent d'entendre des " soutiens " verbaliser leurs propres attentes : « [...] parfois, j'ai l'impression que les gens aident en voulant bien faire, mais attendent en retour quelque chose " (membre du comité de soutien, entretien, 23 février 2018). Néanmoins, la relation d'aide établie amène les volontaires à porter un regard réflexif sur leurs actions et semble rétablir ainsi une forme de réciprocité :

[...] ils nous remercient tout le temps pour ce qu'on fait, mais je leur ai dit que ce n'est pas à sens unique parce qu'on ne fait rien par hasard et gratuitement. [...] La VSP m'aide à titre personnel dans ma vie et $\mathrm{m}^{\prime}$ apporte quelque chose qui me fait sentir utile, donc je leur dis merci aussi. (membre du comité de soutien, entretien, 23 février 2018)

Le bénéfice est ainsi réciproque.

\section{De la proximité à la distance : luttes transversales et autonomisation}

La réciprocité se rétablit aussi lorsque les migrants s'engagent dans des actions qui sortent des objectifs spécifiques du collectif et visent la revendication de droits de catégories transversales d'acteurs sociaux (travailleurs, jeunes, hommes et femmes, pensionnés, etc.) "victimes d'exclusion" (membre de la VSP, notes de terrain, 22 novembre 2017). Faire converger les luttes favorise en outre le passage de l'action humanitaire à l'action politique dans un contexte idéologiquement connoté qui " unit » 
des individus dans un mouvement portant une ou plusieurs " causes " communes (ancien membre de la VSP, entretien, 31 janvier 2018).

Néanmoins, cette proximité avec le contexte et les revendications de ses acteurs est remise en question lorsqu'une situation de dépendance semble apparaître et contraindre l'action autonome des migrants, associés irrémédiablement à une situation de besoin :

[...] j'étais écœuré qu'ils disent "les pauvres, les pauvres », c'est toujours nous les pauvres. Quand on est dans un besoin on peut demander de l'aide [...], mais l'aide doit amener à la libération. (ancien membre de la VSP, entretien, 31 janvier 2018)

La réorientation politique de l'action humanitaire va de pair avec la mise en réseau d'acteurs professionnalisés et une restructuration de la VSP visant la désignation d'un porte-parole des intérêts du collectif ; le but étant d'établir, en principe, une égalité dans l'échange entre membres de la VSP et membres du comité de soutien, ainsi qu'un équilibre de voix dans les négociations avec les autorités et autres interlocuteurs politiques. Pourtant, des conflits ont émergé dans cette répartition des rôles. Le premier porte-parole, parmi les fondateurs du mouvement et en relation avec plusieurs associations locales, s'est par la suite retiré à cause de tensions internes ${ }^{17}$, laissant vide le rôle de représentant du mouvement : « [...] ceux qui n'ont pas de plume, pas de voix, qui va parler pour eux ? " (ancien membre de la VSP, entretien, 30 janvier 2018). Se sentant faiblement représentés dans le rapport de force entre les différents acteurs des négociations, certains membres de la VSP ont considéré l'intervention des "soutiens " nécessaire dans la concertation politique, estimant ne pas pouvoir assumer seuls les revendications : "Nous on ne savait pas, on n'a pas de contacts. On a la volonté de mener la lutte, et les structures sont derrière nous " (membre de la VSP, notes de terrain, 22 novembre 2017). Le manque d'autonomie dérive d'une situation de déséquilibre de pouvoir qui semble se perpétuer en dépit des tentatives de la renverser; situation dénoncée par certains migrants comme une forme de "néocolonialisme contemporain ", résultant de faits historiques qui impactent les relations actuelles:

[...] la colonisation a été le plus grand crime contre l'humanité que l'Occident ait commis, à mes yeux. Non seulement on nous a colonisés, mais on nous a aliénés. Pour chaque Africain, tout ce qu'un Blanc dit est vrai. [...] Quand je regarde [l'occupation] aujourd'hui, j'ai juste envie de pleurer. [...] Ce qu'on a fait au sein de la VSP, c'est exactement une reproduction de ce qu'on fait dans les pays du Sud. (ancien membre de la VSP, entretien, 30 janvier 2018)

Dans cette optique, le rôle joué par le comité de soutien est interprété comme la perpétuation d'un système de dépendance socio-économique, culturelle et politique, au sein duquel l'humanitaire correspondrait à la défense d'intérêts économiques des associations : " chacun vient pour défendre ses subsides en fait ${ }^{18}$ " ; ce qui pérennise une

${ }^{17}$ Ces conflits concernaient principalement le relogement des femmes et enfants dans un nouveau bâtiment, séparé des autres membres de la VSP (hommes et femmes sans enfants), et la disparité perçue dans le soutien aux intérêts de chacun des deux sous-groupes du collectif.

${ }^{18}$ La référence est aux financements reçus pour le développement de projets qui engagent les migrants. 
situation d'exclusion et de subordination, au détriment des intérêts du collectif : " on s'en fout qu'ils aient leurs papiers ou pas » (ancien membre de la VSP, entretien, 30 janvier 2018). La délégation de la prise de parole entraverait l'atteinte de l'autonomie des individus concernés, mettant en péril la conscience politique collective du groupe : " [les "soutiens"] veulent qu'on soit dépendants. [...] En fait la lutte des sans-papiers a été prise en tenaille " (ancien membre de la VSP, entretien, 30 janvier 2018). La rhétorique de la "transversalité des luttes" agirait ici non plus comme moyen de rétablir la réciprocité dans la relation d'aide, mais plutôt comme processus de récupération des intérêts des migrants, lesquels seraient noyés dans des objectifs plus larges : " II ne faut pas que la VSP serve à asseoir une [autre] lutte politique" (membre du comité de soutien, entretien, 23 février 2018). De là vient la volonté d'un positionnement politique propre et d'une prise de distance de l'aide humanitaire : "Moi je viens pour parler de papiers et vous me dites "est-ce qu'ils ont mangé ?!" » (ancien membre de la VSP, entretien, 30 janvier 2018). La démarche politique de la VSP vise la reconnaissance des droits des individus résidant sur le territoire, indépendamment de leur statut juridique, dans une perspective de gestion inclusive des migrations. Lorsqu'en 2016 la ville a vendu les bâtiments de l'occupation à un acheteur privé qui a annoncé par la suite sa volonté de reprendre possession de ses locaux, la situation du collectif est devenue encore plus précaire. La question du logement est passée d'un "simple " besoin de base en un objet de la négociation collective avec les autorités locales. Les revendications formulées convergent vers une demande de régularisation ${ }^{19}$ de personnes résidant sur le territoire depuis longtemps, qui ont fait de la ville de Liège leur espace de vie et de participation socioculturelle et économique, en dépit de l'absence d'un statut de séjour légalement reconnu. Ces démarches ont également un objectif de plus grande portée, car elles visent une remise en question des politiques migratoires contemporaines, auxquelles « il faut qu'on dise "stop" " (membre de la VSP, notes de terrain, 22 novembre 2018). Certains membres de la VSP dénoncent notamment le fait que « [...] la politique d'externalisation des frontières s'adresse surtout aux migrants africains " (membre de la VSP, notes de terrain, 20 avril 2018), mettant ainsi en évidence un dispositif du processus de racialisation et de subalternisation des migrants subsahariens (De Genova, 2018) dont ils font l'expérience concrète.

\section{Conclusion}

Dans un contexte de " crise " où se confrontent les discours sécuritaires et humanitaires, les initiatives citoyennes venant en soutien aux migrants à Bruxelles et à Liège ont joué un rôle important tant dans l'aide aux besoins de base des migrants que dans la contestation des politiques jalonnant leurs parcours migratoires. Ces mouvements citoyens, qui se développent autour d'un positionnement moral empreint d'empathie et de compassion, participent dans leurs actions comme dans leurs effets à une remise en

${ }_{19}$ Par le passé, des campagnes de régularisation one-shot ont permis l'obtention d'un permis de séjour de la part de personnes se trouvant dans des situations similaires dans la ville. 
question des discours sécuritaires. Ce faisant, ces initiatives humanitaires génèrent - et se chevauchent à - une démarche politique orientée vers la contestation des dispositifs de gouvernance des migrations, et plus spécifiquement les politiques d'immigration, d'accueil et de régularisation.

La réorientation politique des initiatives humanitaires se fait, comme nous l'avons démontré dans les deux cas d'étude, à travers une redéfinition des relations interpersonnelles. Les déséquilibres de départ sont questionnés et les acteurs concernés proposent des formes concrètes de rétablissement de la réciprocité - que ce soit les volontaires ou les migrants qui initient ce processus. Les matériaux collectés au sein des deux terrains de recherche, qualitativement différents en termes de profils des acteurs concernés et d'actions menées, illustrent un même processus de réorientation politique. La situation initiale, séparant ceux qui "soutiennent", qui aident, qui donnent, de ceux qui sont soutenus, aidés et qui reçoivent, est questionnée et favorise l'émergence de formes de politisation des mouvements humanitaires. Les migrants "sans-papiers ", démunis de droits et de capacité d'action car mis à l'écart à la fois par les politiques migratoires et par le régime humanitaire en tant que dispositif de gouvernementalité, peuvent reprendre une place active dans les initiatives en question.

Des stratégies diverses, allant de la négociation de la réponse fournie aux attentes des hébergeurs de la Plateforme de soutien aux réfugiés de Bruxelles, jusqu'à la revendication d'une lutte politique autonome chez les membres de la Voix des sans-papiers de Liège, permettent aux migrants dans les deux terrains analysés de rétablir leur agentivité et d'amener par deux processus profondément différents l'action humanitaire dans le registre du politique. Il s'agit aussi de la création d'espaces de prise de parole, ou de délégation de celle-ci, en mettant d'une certaine manière l'histoire migratoire de chacun au service de la mobilisation politique. Si ce processus se développe au sein d'un champ de forces où les rôles ne sont a priori pas égalitaires, l'analyse au niveau micro des relations interpersonnelles témoigne d'une dynamique constructive et tangible de réappropriation de droits et de construction de subjectivités politiques.

\section{Bibliographie}

Agamben G., 1998, Homo Sacer: Sovereign Power and Bare Life, Stanford, Stanford University Press.

Agier M., 2008, Gérer les indésirables. Des camps de réfugiés au gouvernement humanitaire, Paris, Flammarion.

Atlani-Duault L., Dozon J.-P., 2011, "Colonisation, développement, aide humanitaire. Pour une anthropologie de l'aide internationale ", Ethnologie française, $\mathrm{n}^{\circ}$ 41, pp. 393-403.

Brennan D., 2018, « Undocumented People (En)Counter Border Policing: Near and Far from the US Border ", Migration and Society, $\mathrm{n}^{\circ} 1$, pp. 156-163.

De Genova N., 2018, "The "migrant crisis" as racial crisis: do Black Lives Matter in Europe? », Ethnic and Racial Studies, $\mathrm{n}^{\circ} 41$, pp. 1765-1782.

Deleixhe M., 2018, « L'événement de la rencontre », Esprit, n 7, pp. 130-138. 
Fassin D., 2007, « Humanitarianism as a Politics of Life », Public Culture, n 19, pp. 499-520.

Fassin D., 2010, La raison humanitaire : une histoire morale du temps présent, Paris, Gallimard.

Harrell-Bond B., 2002, "Can humanitarian work with refugees be humane? ", Human Rights Quarterly, $\mathrm{n}^{\circ} 24$, pp. 51-85.

Heins V.M., Unrau C., 2018, « Refugees welcome: Arrival gifts, reciprocity, and the integration of forced migrants ", Journal of International Political Theory, n 14, pp. 223-239.

Isin E.F., Nielsen G.M. (eds.), 2013, Acts of Citizenship, London, Zed Books.

Kosnick K., 2019, "New Year's Eve, Sexual Violence and Moral Panics », in J.J. Bock, S. Macdonald (eds.), Refugees Welcome? Difference and Diversity in a Changing Germany, New York, Oxford, Berghahn Books, pp. 171-190.

Martiniello M., Rea A., 2014, " The concept of migratory careers: Elements for a new theoretical perspective of contemporary human mobility », Current Sociology, n 62, pp. 1079-1096.

Mauss M., 1923, "Essai sur le don forme et raison de l'échange dans les sociétés archaïques ", L'année sociologique (1896/1897-1924/1925), pp. 30-186.

Médecins sans frontières, 2019, « Une fuite sans fin : soins en santé mentale au hub humanitaire de Bruxelles », rapport, Bruxelles, Médecins sans frontières, 28 p.

Nussbaum M.C., 2018, The Monarchy of Fear. A Philosopher Looks at our Political Crisis, New York, Simon \& Schuster.

Olivier de Sardan J.-P., 1975, "Afrique : qui exploite qui ? », Les Temps modernes, $\mathrm{n}^{\circ}$ 346, pp. 15061551, n³47, pp. 1774-1775.

Pandolfi M., 2000, « Une souveraineté mouvante et supracoloniale », Multitudes, n 3, pp. 97-105.

Phillimore J., Humphris R., Khan K., 2018, " Reciprocity for new migrant integration: resource conservation, investment and exchange ", Journal of Ethnic and Migration Studies, $\mathrm{n}^{\circ} 44$ pp. 215-232.

Rosenberger S., Stern V., Merhaut N., 2018, Protest movements in asylum and deportation, Cham, Springer International Publishing.

Siméant J., 1998, La cause des sans-papiers, Paris, Presses de Sciences Po.

Tazzioli M., Walters W., 2019, "Migration, solidarity and the limits of Europe », Global Discourse, $\mathrm{n}^{\circ}$ 9, pp. 175-190.

Ticktin M., 2006, "Where Ethics and Politics Meet: The Violence of Humanitarianism in France », American Ethnologist, $\mathrm{n}^{\circ} 33$, pp. 33-49.

Valluy J., 2009, Rejet des exilés : le grand retournement du droit d'asile, Paris, Éditions du Croquant.

Vandervoordt R., Verschraegen G., 2019, « Subversive Humanitarianism and Its Challenges: Notes on the Political Ambiguities of Civil Refugee Support ", in M. Feischmidt, L. Pries, C. Cantat (eds.), Refugee Protection and Civil Society in Europe, Cham, Palgrave Macmillan, pp. 101-128. 